\section{RMD Open}

Rheumatic \&

Musculoskeletal Diseases

\title{
Fluorescence optical imaging: ready for prime time?
}

\author{
Sarah Ohrndorf (DD , ${ }^{1}$ Anne-Marie Glimm, ${ }^{1}$ Mads Ammitzbøll-Danielsen (D) ,2,3 \\ Mikkel Ostergaard, ${ }^{2,3}$ Gerd R Burmester (i) ${ }^{1}$
}

To cite: Ohrndorf S, Glimm A-M, Ammitzbøll-Danielsen $\mathrm{M}$, et al. Fluorescence optical imaging: ready for prime time?. RMD Open 2021;7:e001497. doi:10.1136/ rmdopen-2020-001497

- Additional online supplemental material is published online only. To view, please visit the journal online (http://dx.doi.org/10.1136/ rmdopen-2020-001497).

Received 26 February 2021 Accepted 22 May 2021

\section{Check for updates}

(C) Author(s) (or their employer(s)) 2021. Re-use permitted under CC BY-NC. No commercial re-use. See rights and permissions. Published by BMJ.

${ }^{1}$ Department of Rheumatology and Clinical Immunology, Charité - Universitätsmedizin Berlin, Campus Mitte, Humboldt - Universität zu Berlin, Freie Universität Berlin, Berlin, Germany

${ }^{2}$ Copenhagen Center for Arthritis Research, Center for Rheumatology and Spine Diseases, Rigshospitalet, Copenhagen, Denmark ${ }^{3}$ Department of Clinical Medicine, University of Copenhagen, Copenhagen, Denmark

Correspondence to Dr Gerd R Burmester; gerd.burmester@charite.de

\section{ABSTRACT}

The novel technique of fluorescence optical imaging (FOI, Xiralite), which is approved in the European Union and the USA for clinical use, has been the object of studies since 2009. Indocyanine green-based FOI can demonstrate an impaired microcirculation caused by inflammation in both hands in one examination. Several studies have investigated FOl for detection of joint inflammation by comparing FOI to magnetic resonance imaging (MRI) and/ or musculoskeletal ultrasound (MSUS). The results have shown a generally good agreement $(>80 \%)$ between FOI and clinical examination, MRI and MSUS by power Doppler in inflammatory joint diseases. Moreover, characteristic enhancements in skin and nails are seen in PsA, which potentially can be useful in the diagnostic process of early undifferentiated arthritis. Furthermore, FOI has been investigated for the visualisation of a disturbed microcirculation in the hands and fingers of patients with systemic sclerosis (SSc), highlighting the potential of monitoring vascular changes in SSc and other vasculopathies. The available data indicate that it is time to consider FOI as a useful part of the imaging repertoire in rheumatology clinical practice, particularly where MSUS and MRI are not easily available.

\section{INTRODUCTION}

With new therapeutic options for treating rheumatic inflammatory joint diseases to target, early diagnostic procedures have become increasingly important. In modern rheumatology, the process of establishing an early diagnosis may be greatly helped by imaging procedures. Thus, magnetic resonance imaging (MRI) and musculoskeletal ultrasound (MSUS) with their advantages such as valid detection of inflammation with high sensitivity and good interreader reliability and disadvantages such as costs, contraindications and dependence on the examiner are already established procedures. In recent years, novel imaging techniques have also been developed including the new technique of fluorescence optical imaging (FOI, Xiralite) that has been the object of studies since 2009, and is approved in the European Union and the USA for clinical use.

\section{Key messages}

The novel indocyanine green-based fluorescence optical imaging (FOI) is a fast, easy, and welltolerated method to visualize a disturbed microcirculation in both hands.

- FOI demonstrates an enhanced microcirculation in the joints of both hands due to inflammation caused by various rheumatic joint diseases.

- Characteristic enhancements in skin and nails of both hands are visualized by FOI, especially in psoriatic arthritis.

- FOI presents a reduced microcirculation in systemic sclerosis, which is associated with digital ulcer development.

This comprehensive review will focus on FOI and address the question if it is ready for clinical use. We believe that FOI can complement our current imaging repertoire.

\section{HOW DOES FOI WORK?}

The basis of FOI in rheumatology is the visualisation of an impaired microcirculation in the joints of both hands, which is caused by an inflammatory process due to vasodilatation, hypervascularisation, increased capillary permeability and neoangiogenesis. Following intravenous application of indocyanine green (ICG) as fluorescence-optical dye, disturbed microcirculation in the hands is visualised via a connected software. The principle of ICG usage was extensively investigated by Cherrick $e t a l .{ }^{1}$ Today, it is used in ophthalmology, cardiology and gastroenterology and was there demonstrated to be a well-known and well-tolerated dye. A disadvantage of FOI is the invasiveness of the examination because of the need of an intravenous access. In addition, kidney and liver function should usually be within normal limits and hyperthyroidism must be excluded.

The intensity of the dye concentration is displayed via a false-colour grading: white as a very high concentration and intensity 
followed by red, yellow and green in descending order. The examination lasts $6 \mathrm{~min}$ recording one image per second and adding up to a total cluster of 360 images.

Advantages of this method are the high acceptance of the patients and the possibility to examine all joints of both hands and wrists (30 joints in total) within the described short period of time of $6 \mathrm{~min}$. FOI is a delegable task that can be performed by a medical assistant or nurse with a physician in the background. Accordingly, the images can be evaluated at remote sides. The acquisition costs of an FOI device are comparable with a high-end ultrasound machine and less expensive than an MRI machine.

Efforts have been made by different research groups to standardise the FOI image analysis. The comparison of three scoring methods from Berlin, Stockholm and Copenhagen showed moderate to good inter-reader reliability for all three FOI scoring methods (Pabak-OS: 0.50-0.78, ICC: $0.43-0.85)$ used in patients with erosive hand osteoarthritis (OA) and rheumatoid arthritis (RA) with similar sensitivities $(63 \%-65 \%)$ and specificities (76\%-91\%) compared with MRI. ${ }^{2}$ Mostly, the 'Berlin scoring method' is used including three phases dependent on the blood flow of the fingertips and the automatically generated sum image (PrimaVistaMode, PVM). The evaluation of the inflammatory intensity in the phases 1-3 and PVM is performed by a semiquantitative (0-3) score called FOIAS (FOI Activity Score).$^{2-13}$ Recently, the 'Copenhagen scoring method' FOIE-GRAS (FOI Enhancement-Generated RA Score) has been published, which presents a feasible, reliable and responsive single score for synovitis assessment in RA. ${ }^{14}$

\section{PRECLINICAL AND ANIMAL STUDIES ON FOI}

Fischer et al evaluated in vivo fluorescence imaging of experimental inflammatory joint disease in a model of Borrelia-induced Lyme arthritis presenting that the fluorescence signal differed significantly between controls and arthritic animals $(\mathrm{p}<0.05) .{ }^{15}$ Meier et al evaluated a combined X-ray/optical imaging system for ICGenhanced detection of arthritic joints in a rat model of antigen induced arthritis of the knee and ankle, which were compared for significant differences with controls. The fluorescence signal of arthritic joints was significantly higher compared with the non-arthritic control joints $(\mathrm{p}<0.05) .{ }^{16}$ Moreover, Vollmer et al described an in vivo near-infrared (NIR) fluorescence imaging technique for therapy monitoring of ankle joints affected by collagen-induced arthritis before and after treatment demonstrating a statistically significant decrease in fluorescence intensity in ankle joints $(\mathrm{p}<0.05) .{ }^{17}$

\section{WHAT IS THE CURRENT EVIDENCE OF FOI IN RHEUMATOLOGY?}

Different research groups all over the world have examined FOI in numerous studies in the last 10 years for different rheumatologic diseases and indications; please see online supplemental table 1 in which full papers

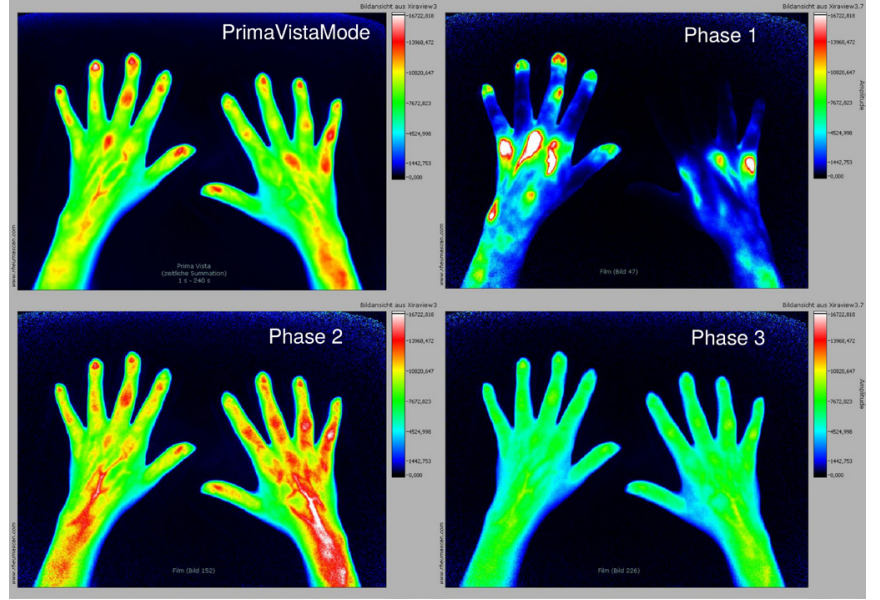

Figure 1 FOl images in PrimaVistaMode and phases 1-3 in a patient with active, early seropositive rheumatoid arthritis. Early and strong enhancements within MCP joints in phase 1. Furthermore, moderate enhancement of grade 2 within right PIP joints in phase 2. FOI, fluorescence optical imaging; $\mathrm{MCP}$, metacarpophalangeal; PIP, proximal interphalangeal.

(congress abstracts excluded) are listed according to their order in the text.

\section{Inflammatory joint diseases}

Most notably, the validity studies in patients with inflammatory rheumatoid joint diseases have shown a mostly good agreement between FOI, clinical examination (CE), MSUS and MRI.

Fischer et al presented first clinical data on five patients with RA and a corresponding number of volunteers that were examined using fluorescence imaging in the NIR spectral range following the intravenous administration of an unspecific contrast agent with ICG. 0.2 Tesla (T) MRI served as the reference method. In this study, inflammatory joints of RA patients were enriched by ICG and showed a different kinetic behaviour compared with normal joints presenting the capability of contrastenhanced fluorescence imaging to detect early inflammatory changes with good correlation to MRI $(\mathrm{r}=0.84) .{ }^{18} \mathrm{In}$ a subsequent study including 252 patients with different forms of arthritis, Werner et al demonstrated a sensitivity of $76 \%$ and a specificity of $54 \%$ for FOI in detecting joint inflammation (synovitis/tenosynovitis)—compared with MRI. Here, FOI in phase 1 had a high specificity of $94 \%$. Furthermore, FOI showed agreement rates up to $88 \%$ vs CE, $64 \%$ vs MSUS in grey scale, $88 \%$ vs MSUS in power Doppler and $83 \%$ vs MRI, depending on the compared phase and parameter. ${ }^{3}$ In another study by Werner et al including 32 patients with early and very early arthritis as well as 46 controls, the sensitivity of FOI was $86 \%$ and the specificity was $63 \%$, while the sum image (PVM), phase 1 and phase 3 reached high specificities of $87 \%, 90 \%$ and $88 \%$, respectively, compared with MRI. ${ }^{4}$ In contrast, Meier et al reported that FOI had a sensitivity of only $39.6 \%$ and a specificity of $85.2 \%$, compared with $3.0 \mathrm{~T}$ MRI. In this study, 45 patients with clinically suspected inflammatory 


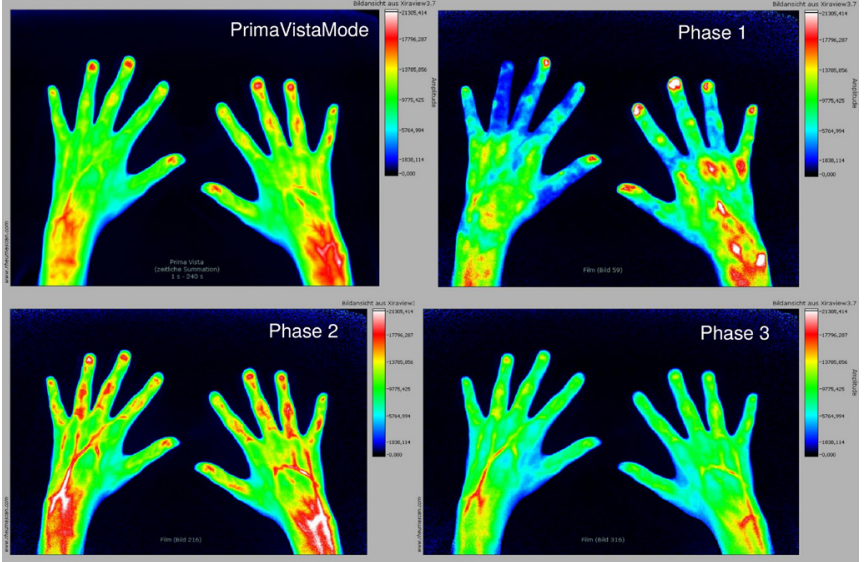

Figure 2 FOl images in PrimaVistaMode and phases 1-3 in a patient with clinically inactive (DAS28 <2.6) rheumatoid arthritis. Apart from clinical presentation, FOI shows early enhancement within right wrist and MCP joints in phase 1. DAS28, Disease activity score 28; FOI, fluorescence optical imaging; MCP, metacarpophalangeal.

arthropathy were included. Referring to the reduced sensitivity, the authors stated that FOI showed limitations for the detection of inflamed joints of the hand in comparison with MRI. ${ }^{19}$ In contrast, Krohn et al examined 31 early RA patients via FOI-compared with MSUS in grey scale and power Doppler as well as 0.31T MRI. The working group presented an overall sensitivity/specificity for FOI of $81 \% / 0 \%, 49 \% / 84 \%$ and $86 \% / 38 \%$ for wrist, metacarpophalangeal (MCP) and proximal interphalangeal (PIP) joints, respectively, in comparison to MRI, and an overall sensitivity/specificity for FOI of $88 \% / 15 \%$, $81 \% / 76 \%$ and $100 \% / 27 \%$ for wrist, MCP and PIP joints, respectively-compared with power Doppler US. FOI in phase 2 was the most sensitive phase, while phase 1 showed the highest specificity, ${ }^{5}$ comparable to the results by Werner et $a l^{3}{ }^{4}$ An FOI image example of an active, early RA patient is given by figure 1 . In addition, Schäfer et al analysed 90 individual joints from 18 patients with

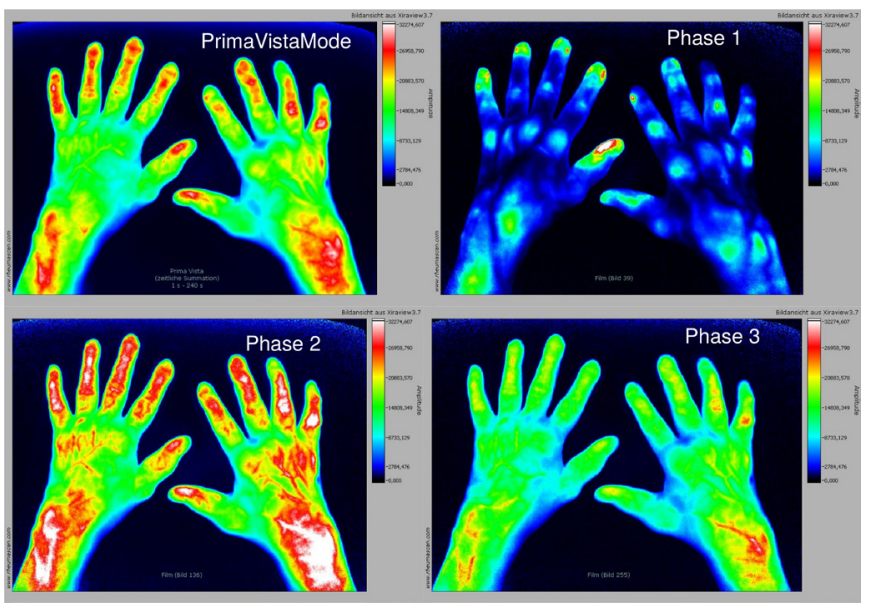

Figure 3 FOl images in PrimaVistaMode and phases 1-3 in a patient with longstanding, active rheumatoid arthritis. FOI, fluorescence optical imaging.
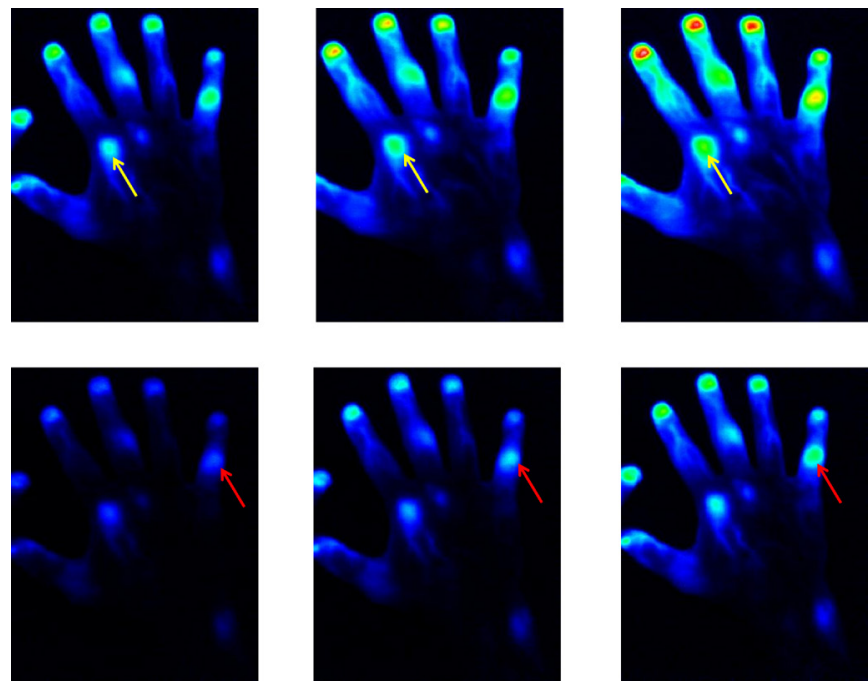

Figure 4 FOIE-GRAS images in a patient with RA showing enhancements in MCP2 (yellow arrows) and PIP5 joints (red arrows) at different time points. FOIE-GRAS, Fluorescence Optical Imaging Enhancement-Generated rheumatoid arthritis Score; MCP, metacarpophalangeal; PIP, proximal interphalangeal; RA, rheumatoid arthritis.

active RA via FOI and found a sensitivity of 26/39 (67\%) and a specificity of $31 / 40(77 \%)$ for fluorescence ratio of phase 3 using a cut-off value of more than 1.2 to detect 1.5T MRI-confirmed synovitis with FOI. They concluded that FOI has a potential for visualising synovitis in subjects with RA. ${ }^{20}$ Subclinical synovitis as clinically non-apparent but visible pathology in MSUS could also be detected by FOI with a sensitivity of $80 \%$ and specificity of $96 \%$. Thus, Kisten et al suggested the potential use of FOI for the screening of synovitis and clinically inactive inflammatory joint disease (see figure 2 as an FOI example of a clinically inactive (DAS28 <2.6) RA patient), especially when MSUS is not available. ${ }^{21}$ Thuermel et al examined 20 patients with highly active RA and 13 healthy volunteers by CE, FOI and contrast-enhanced 3.0T-MRI and

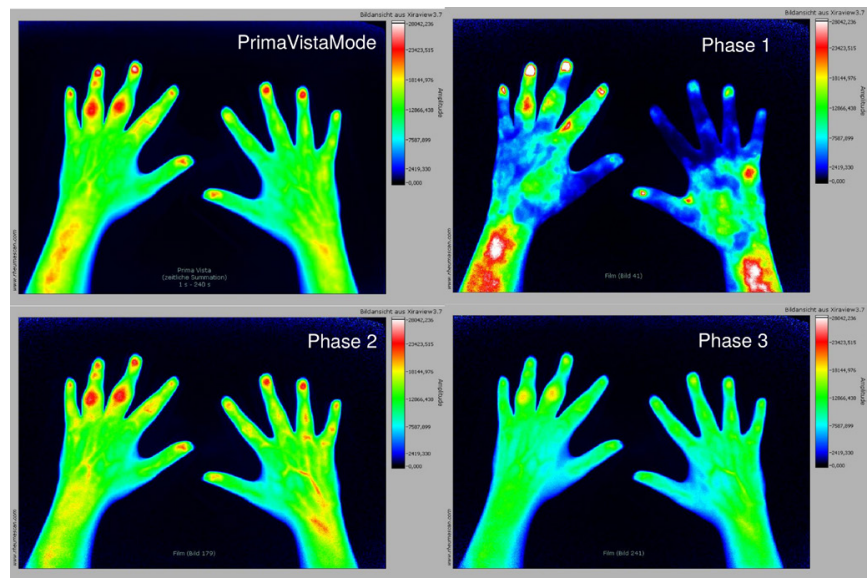

Figure 5 FOl images in PrimaVistaMode and phases 1-3 in a patient with active psoriatic arthritis. Especially, visualised strong and early as well as reamaining enhancement in PIP3 and PIP4 joints of the left hand. FOI, fluorescence optical imaging; PIP, proximal interphalangeal. 


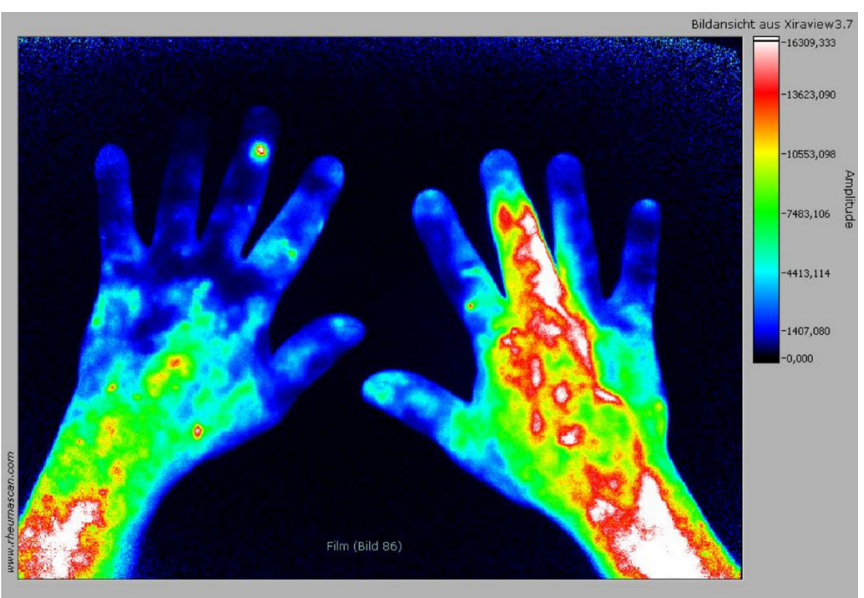

Figure 6 FOI image in a patient with active psoriatic arthritis with dactylitis (third right finger) and subclinical skin enhancement, especially on the dorsum of the right hand. FOI, fluorescence optical imaging.

reported an overall sensitivity of $57.3 \%$ and specificity of $92.1 \%$ of FOI versus MRI for the detection of synovitis. In addition, they demonstrated that the sensitivity of FOI increased with the degree of synovitis to $65.0 \%$ for moderate and severe synovitis (specificity $88.1 \%$ ) and $76.3 \%$ for severe synovitis (specificity $80.5 \%$ ), while FOI's performance decreased with the degree of synovitis with false negative results predominantly for mild (45.5\%) and moderate $(46.6 \%)$ synovitis and false positive results predominantly based on mild (grade 1) signals $(81.6 \%$ ). Accordingly, the authors concluded that FOI has a lower sensitivity than 3.0T-MRI and its diagnostic performance decreases with the degree of synovitis correspondent to the strength of FOI signals. ${ }^{22}$ Hirano et al compared performance profiles of CE, MSUS and FOI using $1.5 \mathrm{~T}$ MRI as a reference in six active RA patients. FOI showed sensitivities and specificities of $85 \%$ and of $94 \%$ (phase
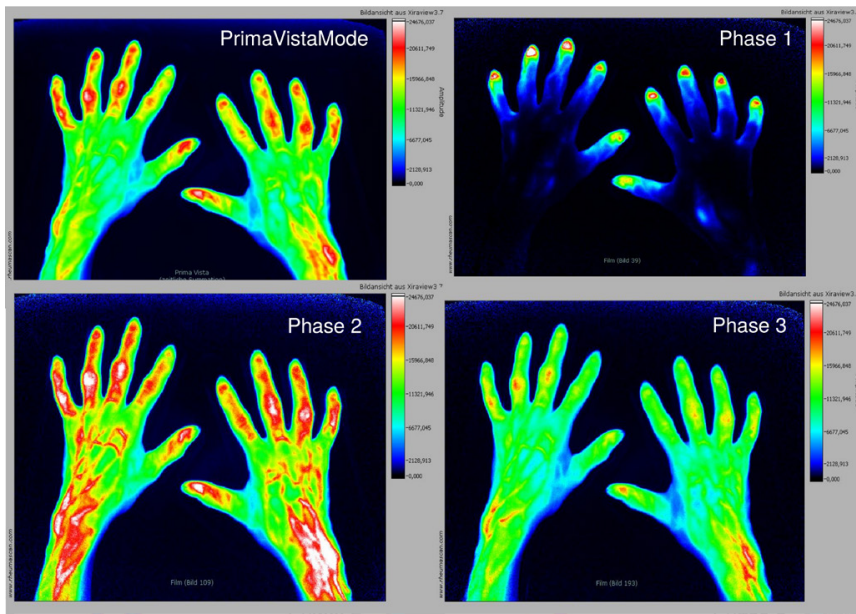

Figure 7 FOl images in PrimaVistaMode and phases 1-3 in a patient with osteoarthritis. Visible streaky enhancement in PIP2 joints of both hands. Additionally, strong enhancment in phase two and PVM in PIP3-5 joints as sign for active OA. FOI, fluorescence optical imaging; OA, osteoarthritis; PIP, proximal interphalangeal.

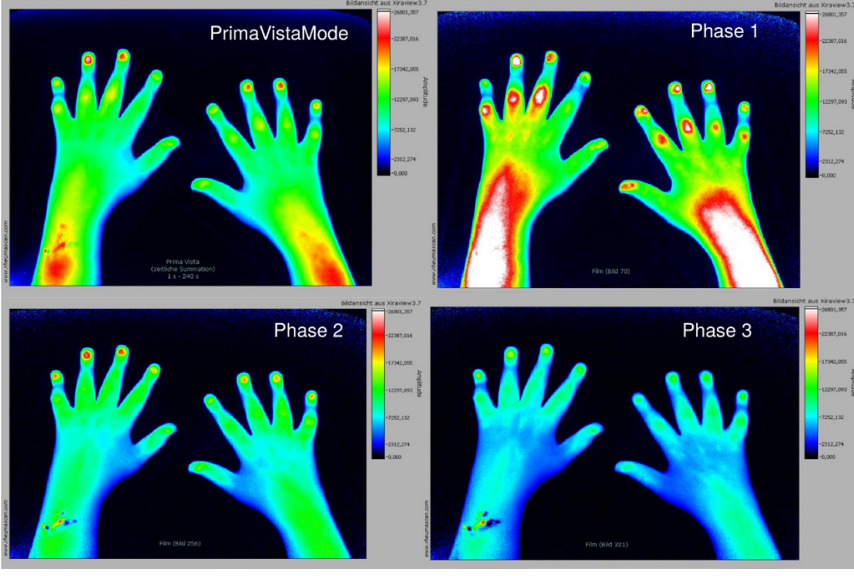

Figure 8 FOl images in PrimaVistaMode and phases 1-3 in a patient with active juvenile idiopathic arthritis showing enhancements in PIP joints in phase 1. FOI, fluorescence optical imaging; PIP, proximal interphalangeal.

1), and $69 \%$ and $94 \%$ (phase 2) in the detection of synovitis, which were comparable to those of MSUS and more specific than CE. They concluded that FOI has a potential as an assessment modality of RA. ${ }^{12}$ An FOI image example of a longstanding, active RA is given by figure 3. Recently, Kawashiri et al explored the significance of the FOI findings based on the association between the FOI and MSUS findings and serum biomarkers (including vascular endothelial growth factor) in 50 consecutive patients with active RA. They presented FOI to be highly sensitive in the detection of synovitis, however, the frequency of positive findings and the diagnostic performance with MSUS as the reference standard for FOI differed considerably among the phases of FOI as well as among the affected joint regions. Furthermore, the FOI scores were positively correlated with clinical disease activity, MSUS scores and serum biomarkers. Moreover, the severity of FOI-proven synovitis was associated with the presence of MSUS-proven bone erosion. ${ }^{13}$ Recently, AmmitzbøllDanielsen et al presented a moderate agreement of FOI (using the FOIE-GRAS method; figure 4) with ultrasound (ICC 0.30-0.54) for total score and moderate correlation with clinical joint assessment and Disease activity score 28 (DAS28)-CRP in RA patients . ${ }^{14}$

In a study on patients with suspected or confirmed psoriatic arthritis (PsA) published by Erdmann-Keding et $a$, FOI was more sensitive than MSUS for the detection of inflammation in PIP/distal interphalangeal (DIP) joints $(p=0.035)$ and different findings of pathologic enhancement for confirmed (pathologic signal enhancement mainly in FOI phase $2+$ phase 3 ) and suspected PsA (pathologic signal enhancement mainly in FOI phase 1) were found, which may be relevant for determining the inflammatory status. ${ }^{9}$ An FOI image in a patient with active PsA is demonstrated in figure 5.

Apart from the inflammatory joint changes, Schmidt et al investigated inflammatory enhancements in the skin area of both hands that were observed in patients with psoriasis vulgaris $(\mathrm{PsO})$ and $\mathrm{PsA}$ compared with 
RA patients and healthy controls in a retrospective analysis. Subclinical skin enhancement on the back of the hands was more common in PsO/PsA $(72.5 \%)$ than in RA patients $(20.5 \%)$ and healthy individuals $(28.0 \%)$ $(\mathrm{p}<0.001)$. Based on the FOI pattern, most patients with $\mathrm{PsO} / \mathrm{PsA}(72.5 \%)$, RA (76.9\%), and healthy controls $(68.0 \%)$ were classified correctly, which indicate FOI's potential to study microcirculation in rheumatic diseases with skin involvement. ${ }^{23}$ An FOI image example of a patient with active PsA (dactylitis) and skin enhancement is given by figure 6 . Moreover, the 'green nail' phenomenon has been described by Wiemann $e t a l$ as a possible microcirculation disturbance in the area of the nail with a specificity of $87 \%$ for PsA in relation to an RA control cohort. ${ }^{24}$ Werner et al had described a triangular, slightly arcuate enhancement from the nail bed into the DIP that was observed in 60 out of $64(94 \%)$ subjects with PsA compared with only 8 out of $38(21 \%)$ patients with definite RA. ${ }^{3}$

\section{Osteoarthritis}

In a subsequent study by Glimm et al, the amount and distribution of inflammatory signs in wrist and finger joints via FOI and MSUS was compared in RA vs OA patients. FOI and MSUS detected inflammation in both RA and OA highlighting the inflammatory component in the course of OA. The authors assumed that the different inflammatory patterns and various shapes of fluorescence enhancement may offer opportunities to distinguish both diseases via FOI. ${ }^{6}$ An FOI image example in a patient with $\mathrm{OA}$ is given by figure 7 . In contrast, a study by Maugesten et al of 221 hand OA patients showed limited correlations between FOI, MRI and MSUS, questioning the value of FOI for the detection of synovitis in hand OA. ${ }^{7}$ In another publication by Maugesten et al it was investigated whether FOI enhancement and MRI-defined synovitis were associated with pain and physical function in hand OA. The authors found that FOI enhancement and MRI-defined synovitis were associated with pain in the same finger joint (with numerical stronger associations between MRI-defined synovitis and finger joint pain/tenderness). Nevertheless, none of the imaging modalities demonstrated consistent associations with pain, stiffness and physical function on subject level concluding that FOI enhancement was probably associated with more noise than established imaging modalities (MRI and MSUS), furthermore FOI did not show enhancement in the thumb base joints, which are commonly affected by OA. ${ }^{8}$

\section{Juvenile idiopathic arthritis}

FOI can also be used in children, as the dosage of the dye ICG can be adapted to the patients' weight. Apart from the particularly good tolerability, interestingly, the children investigated accepted the procedure and showed interest in this method due to the colourful presentation.

In paediatric patients with inflammatory (juvenile idiopathic arthritis, JIA) and non-inflammatory joint diseases,
FOI was more sensitive for detecting clinically active joints than MSUS in grey scale and power Doppler (GSUS/ PDUS) $(75.2 \%$ vs $57.3 \% / 32.5 \%)$, which was presented by Beck $e t a l$. The predictive value in this study for discrimination between inflammatory and non-inflammatory joint diseases was 0.79 for FOI and $0.80 / 0.85$ for GSUS/PDUS. The authors concluded that FOI may provide an additional diagnostic method in paediatric rheumatology. ${ }^{11}$ Klein et al presented a 24-week observational study in polyarticular JIA in which 37 patients were evaluated clinically, by MSUS (GS/PD) and FOI at baseline, week 12 and week 24. Twenty-four patients started therapy with methotrexate and 13 patients with a biological agent for the first time (etanercept $n=11$, adalimumab and tocilizumab $n=1$ each). Improvement on treatment with either methotrexate or a biologic therapy could be visualised by FOI and MSUS. Both methods could detect clinical but also subclinical inflammation, which was to a higher extent visualised by FOI than MSUS. ${ }^{25}$ An FOI image example in a patient with JIA is given by figure 8 .

\section{Therapeutic monitoring}

In addition to the diagnostic studies on joint diseases mentioned above, studies on therapeutic monitoring were conducted. Among these, Glimm et al included 35 patients with early RA that were followed-up within 1 year after baseline by FOI both in responders and nonresponders according to EULAR response criteria by DAS28. A significant signal reduction in FOI phase 1 was observed, so that an objective evaluation of the therapeutic success appears possible via FOI. ${ }^{10}$ Moreover, Meier et al demonstrated a significant difference in the rates of early enhancement in FOI-as quantitative measurement-after 24 weeks of therapy start or escalation in different inflammatory arthritis patients. They stated a signal reduction in responders $(-21.5 \%, \mathrm{p}<0.001)$ and increase in non-responders $(+10.8 \%, \mathrm{p}=0.075)$ underlining the possibility of a therapy monitoring per FOI. ${ }^{26}$ In a recent study by Ammitzbøll-Danielsen et al a good sensitivity to change (standardised response mean $>0.80$ ) was found. ${ }^{14}$

\section{Systemic sclerosis}

The FOI procedure was also applied to patients with systemic sclerosis ( $\mathrm{SSc}$ ) to detect soft tissue inflammation by ICG enhancement in 19 segments per hand, which was shown to be significantly reduced 7 days after either iloprost or alprostadil therapy from $40.9 \%$ to $24.7 \%$ presented by Pfeil et al. ${ }^{27}$ Furthermore, FOI was investigated for the visualisation of a disturbed microcirculation in the hands and fingers of SSc patients and to link FOI findings to clinical signs of ischaemia such as digital ulcers (DU) and pitting scars, reflecting progressive vasculopathy in SSc. In the results presented by Friedrich et al, $93.6 \%$ of healthy subjects showed initial ICG signals in their fingertips compared with only $78.5 \%$ in limited SSc and $43.2 \%$ in diffuse SSc. Moreover, FOI findings of missing or reduced microcirculation were significantly 
associated with a late capillaroscopic pattern, disseminated SSc features, a diffuse SSc subtype and the presence of digital ulcers or pitting scars in SSc patients. ${ }^{28}$ In the 12 months follow-up study investigating 76 SSc patients, Friedrich et al could show that fingers with pathological staining by FOI at baseline had a higher risk for new ulcer development in the same finger $(p=0.0153)$. Especially, a missing signal of FOI in the right third digit at baseline was associated with the subsequent development of DU. ${ }^{29}$ Recently, this group has introduced a composite score (CIP-DUS-Clinical features, Imaging, Patient historyDigital Ulcer Score) to predict DU in SSc patients also including FOI, which was able to identify all patients at risk of digital ulcers throughout 12 months. ${ }^{30}$

\section{Summary and conclusion}

Several studies have been published for the use of FOI in the detection of joint inflammation comparing the performance of FOI to MRI and/or MSUS. The results presented high sensitivities and specificities for FOI in phase 1 and phase 3 by all studies using FOIAS in inflammatory joint diseases. ${ }^{3-5} 91213$ Thus, phase-specific evaluation by the FOIAS method with particular attention to phase 1 (highest specificity compared with both power Doppler US and MRI) is strongly recommended. This was confirmed by Glimm et al who found an acceptable responsiveness in phase 1 for RA patients $(<2$ years disease duration) initiating or escalating antirheumatic treatment. ${ }^{10}$ Recently, this was confirmed in a new study using FOIE-GRAS (a peak enhancement score) demonstrating a good responsiveness, which was in line with MSUS scores, joint evaluation and DAS28-CRP. ${ }^{14}$ These data support that FOI is an alternative for monitoring of RA patients in clinical trials and practice.

The value of FOI in patients with OA might be limited since nearly no FOI enhancement in the thumb base joints could be detected. Furthermore, FOI did not demonstrate consistent associations with pain, stiffness and physical function on subject level. ${ }^{8}$ However, FOI seems to offer opportunities to distinguish OA vs RA, therefore, it might become a useful tool in differential diagnosis. ${ }^{6}$

Moreover, inflammatory enhancements in the skin area of both hands were detected by FOI indicating its potential to study microcirculation in rheumatic diseases with skin involvement. ${ }^{22}$ This and other microcirculation disturbances ('green nail sign' etc) need to be confirmed by prospective studies on larger scales, but they give first hints that FOI has the potential to present both skin and joint involvement at the same time underlining its potential in patients with PsA for monitoring, for example, objective ('real') psoriatic skin and joint disease stages.

In patients with SSc, a reduced microcirculation in the hands and fingers could be presented by FOI. Furthermore, it could be shown that fingers with pathologic staining detected by FOI at baseline had a higher risk for new ulcer development in the same finger during 12 months. In a composite score called CIP-DUS also including FOI-examination, all SSc patients at risk of digital ulcers throughout 12 months could be predicted. Therefore, FOI also has the potential to be used in the detection of reduced microcirculation in soft tissue diseases.

An advantage of FOI is that a scan of all finger and hand joints is performed within a short time period of $6 \mathrm{~min}$. Furthermore, the procedure can be delegated to a trained nurse-in the presence of a doctor in the background. Due to the weight-dependent application of the dye it is possible to perform FOI also in children. ${ }^{11} 24$ The procedure can be repeated within few hours due to a short half-life of ICG. A disadvantage is the necessary of an intravenous access making FOI an invasive method, but apart from this is a painless method. Also, the use of ICG rarely causes allergic reactions. Thousands of examinations have shown that it is well-tolerated and well-accepted procedure by the patients. We have experienced that patients follow the examination on the screen attentively and that the images enable them to visually understand the disease and to discuss response and non-response to therapy. Limitations of FOI is that the palmar aspects of the hands (eg, flexor tenosynovitis) can only be partly examined due to the limited penetration of infrared light, and that the method provides a twodimensional visualisation of the hand in contrast to MRI and ultrasound. Furthermore, important pitfalls of this method need to be known (eg, wounds located over the joint), which should be limited by a distinct documentation (eg, photographs of both hands).

To summarise, FOI is a fast, easy, and well-tolerated method to visualise a disturbed microcirculation in various rheumatic diseases, and thereby allows for the detection of joint inflammation, vasculopathic changes and skin involvement of the hands.

Therefore, we believe that FOI is a feasible and reliable method which is ready for clinical practice to complement our current imaging repertoire in rheumatology, particularly at sites where US and MRI are not readily available.

\section{Twitter Mads Ammitzbøll-Danielsen @Madsammitzboll}

Acknowledgements We would like to acknowledge the study nurse Gabriela Schmittat for logistical and technical assistance (eg, image acquisition).

Contributors SO, A-MG and GRB contributed substantially to the conception of the review. SO, A-MG and GRB drafted the first version of the review. SO, A-MG, MA-D, M0 and GRB revised the article for important intellectual content. All of the named authors drafted the review and revised it for important intellectual content. All authors gave final approval of the version to be published and agreed to be accountable for all aspects of the work in ensuring that questions related to the accuracy or integrity of any part of the work are appropriately investigated and resolved.

Funding One of the authors (SO) was funded by the 'Advanced Clinician Scientist program' of the German Society of Internal Medicine (Deutsche Gesellschaft für Innere Medizin).

Competing interests None declared.

Patient consent for publication Not required.

Provenance and peer review Commissioned; externally peer reviewed.

Open access This is an open access article distributed in accordance with the Creative Commons Attribution Non Commercial (CC BY-NC 4.0) license, which permits others to distribute, remix, adapt, build upon this work non-commercially, 
and license their derivative works on different terms, provided the original work is properly cited, appropriate credit is given, any changes made indicated, and the use is non-commercial. See: http://creativecommons.org/licenses/by-nc/4.0/.

ORCID iDs

Sarah Ohrndorf http://orcid.org/0000-0001-5943-4688

Mads Ammitzbøll-Danielsen http://orcid.org/0000-0003-4878-0432

Gerd R Burmester http://orcid.org/0000-0001-7518-1131

\section{REFERENCES}

1 Cherrick GR, Stein SW, Leevy CM, et al. Indocyanine green: observations on its physical properties, plasma decay, and hepatic extraction. J Clin Invest 1960;39:592-600.

2 Maugesten Ø., Ohrndorf S, Glinatsi D, et al. Evaluation of three scoring methods for fluorescence optical imaging in erosive hand osteoarthritis and rheumatoid arthritis. Osteoarthr Cartil Open 2020;1:100017.

3 Werner SG, Langer H-E, Ohrndorf S, et al. Inflammation assessment in patients with arthritis using a novel in vivo fluorescence optical imaging technology. Ann Rheum Dis 2012;71:504-10.

4 Werner SG, Langer H-E, Schott P, et al. Indocyanine greenenhanced fluorescence optical imaging in patients with early and very early arthritis: a comparative study with magnetic resonance imaging. Arthritis Rheum 2013;65:3036-44.

5 Krohn M, Ohrndorf S, Werner SG, et al. Near-Infrared fluorescence optical imaging in early rheumatoid arthritis: a comparison to magnetic resonance imaging and ultrasonography. J Rheumatol 2015;42:1112-8.

6 Glimm A-M, Werner SG, Burmester GR, et al. Analysis of distribution and severity of inflammation in patients with osteoarthitis compared to rheumatoid arthritis by ICG-enhanced fluorescence optical imaging and musculoskeletal ultrasound: a pilot study. Ann Rheum Dis 2016;75:566-70.

7 Maugesten Øystein, Mathiessen A, Hammer HB, et al. Validity and diagnostic performance of fluorescence optical imaging measuring synovitis in hand osteoarthritis: baseline results from the Nor-Hand cohort. Arthritis Res Ther 2020;22:98.

8 Maugesten Øystein, Ohrndorf S, Slatkowsky-Christensen B, et al. Associations between fluorescence optical imaging and magnetic resonance imaging and symptoms in hand osteoarthritis. Rheumatology 2021:keab085.

9 Erdmann-Keding M, Ohrndorf S, Werner SG, et al. Fluorescence optical imaging for the detection of potential psoriatic arthritis in comparison to musculoskeletal ultrasound. J Dtsch Dermatol Ges 2019;17:913-21.

10 Glimm A-M, Sprenger LI, Haugen IK, et al. Fluorescence optical imaging for treatment monitoring in patients with early and active rheumatoid arthritis in a 1-year follow-up period. Arthritis Res Ther 2019;21:209.

11 Beck MC, Glimm A-M, Ohrndorf S, et al. Fluorescence optical imaging in pediatric patients with inflammatory and non-inflammatory joint diseases: a comparative study with ultrasonography. Arthritis Res Ther 2017;19:233.

12 Hirano F, Yokoyama-Kokuryo W, Yamazaki H, et al. Comparison of fluorescence optical imaging, ultrasonography and clinical examination with magnetic resonance imaging as a reference in active rheumatoid arthritis patients. Immunol Med 2018;41:75-81.

13 Kawashiri SY, Nishino A, Shimizu T, et al. Fluorescence optical imaging in patients with active rheumatoid arthritis: a comparison with ultrasound and an association with biomarkers. Scand J Rheumatol 2020;21:1-9.
14 Ammitzbøll-Danielsen M, Glinatsi D, Terslev L, et al. A Novel Fluorescence Optical Imaging Scoring System for Hand Synovitis in Rheumatoid Arthritis - validity and agreement with ultrasound. Rheumatology 2021:keab377.

15 Fischer T, Gemeinhardt I, Wagner S, et al. Assessment of unspecific near-infrared dyes in laser-induced fluorescence imaging of experimental arthritis. Acad Radiol 2006;13:4-13.

16 Meier R, Krug C, Golovko D. ICG-enhanced imaging of arthritis with an integrated optical Imaging/X-ray system. Arthritis Rheum 2010;62:2322-7.

17 Vollmer S, Gemeinhardt I, Vater A, et al. In vivo therapy monitoring of experimental rheumatoid arthritis in rats using near-infrared fluorescence imaging. J Biomed Opt 2014;19:036011-7.

18 Fischer T, Ebert B, Voigt J, et al. Detection of rheumatoid arthritis using non-specific contrast enhanced fluorescence imaging. Acad Radiol 2010;17:375-81.

19 Meier R, Thürmel K, Moog P, et al. Detection of synovitis in the hands of patients with rheumatologic disorders: diagnostic performance of optical imaging in comparison with magnetic resonance imaging. Arthritis Rheum 2012;64:2489-98.

20 Schäfer VS, Hartung W, Hoffstetter P, et al. Quantitative assessment of synovitis in patients with rheumatoid arthritis using fluorescence optical imaging. Arthritis Res Ther 2013;15:R124.

21 Kisten Y, Györi N, Af Klint E, et al. Detection of clinically manifest and silent synovitis in the hands and wrists by fluorescence optical imaging. RMD Open 2015;1:e000106.

22 Thuermel K, Neumann J, Jungmann PM, et al. Fluorescence optical imaging and 3T-MRI for detection of synovitis in patients with rheumatoid arthritis in comparison to a composite standard of reference. Eur J Radiol 2017;90:6-13.

23 Schmidt A, Glimm AM, Haugen IK, et al. Detection of subclinical skin manifestation in patients with psoriasis and psoriatic arthritis by fluorescence optical imaging. Arthritis Res Ther 2020;22:192.

24 Wiemann O, Werner SG, Langer $\mathrm{H}-\mathrm{E}$, et al. The "green nail" phenomenon in ICG-enhanced fluorescence optical imaging - a potential tool for the differential diagnosis of psoriatic arthritis. $J$ Dtsch Dermatol Ges 2019;17:138-47.

25 Klein A, Just GW, Werner SG, et al. Fluorescence optical imaging and musculoskeletal ultrasonography in juvenile idiopathic polyarticular disease before and during antirheumatic treatment - a multicenter non-interventional diagnostic evaluation. Arthritis Res Ther 2017;19:147.

26 Meier R, Thuermel K, Noël PB, et al. Synovitis in patients with early inflammatory arthritis monitored with quantitative analysis of dynamic contrast-enhanced optical imaging and MR imaging. Radiology 2014;270:176-85.

27 Pfeil A, Drummer KF, Böttcher J, et al. The application of fluorescence optical imaging in systemic sclerosis. Biomed Res Int 2015;2015:1-6.

28 Friedrich S, Lüders S, Werner SG, et al. Disturbed microcirculation in the hands of patients with systemic sclerosis detected by fluorescence optical imaging: a pilot study. Arthritis Res Ther 2017;19:87.

29 Friedrich S, Lüders S, Glimm AM, et al. Association between baseline clinical and imaging findings and the development of digital ulcers in patients with systemic sclerosis. Arthritis Res Ther 2019;21:96.

30 Friedrich S, Lüders S, Klotsche J, et al. The first composite score predicting digital ulcers in systemic sclerosis patients using clinical data, imaging and patient history-CIP-DUS. Arthritis Res Ther 2020;22:144. 